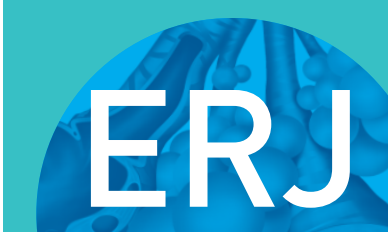

open research

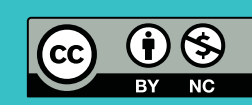

\section{Risk of tuberculosis transmission among healthcare workers}

\author{
Roland Diel (1) ${ }^{1,2}$, Stefan Niemann ${ }^{3,4}$ and Albert Nienhaus ${ }^{5,6}$
}

\begin{abstract}
Affiliations: ${ }^{1}$ Institute for Epidemiology, University Medical Hospital Schleswig-Holstein, Kiel, Germany. ${ }^{2}$ LungClinic Grosshansdorf, Airway Research Center North (ARCN), German Center for Lung Research (DZL), Germany. ${ }^{3}$ Molecular and Experimental Mycobacteriology Group, Reference Center for Mycobacteria, Research Center Borstel, Borstel, Germany. ${ }^{4}$ German Center for Infection Research, Borstel, Germany. ${ }^{5}$ Institute for Health Service Research in Dermatology and Nursing, University Medical Center HamburgEppendorf, Hamburg, Germany. ${ }^{6}$ Institution for Statutory Accident Insurance and Prevention in the Health and Welfare Services (BGW), Hamburg, Germany.
\end{abstract}

Correspondence: Roland Diel, Institute for Epidemiology, University Medical Hospital Schleswig-Holstein, Niemannsweg 11, 24015 Kiel, Germany. E-mail: roland.dieldepi.uni-kiel.de

ABSTRACT Data from a prospective molecular-epidemiological study (1997-2015) of patients with culture-confirmed tuberculosis in Hamburg, Germany, were evaluated to assess the occupational risk of Mycobacterium tuberculosis complex transmission in a low-incidence setting.

Isolates of $M$. tuberculosis complex were genotyped using IS6110 restriction fragment length polymorphism analysis. Results of structured questionnaires, geographical mapping and additional patient interviews were used for confirming epidemiological links.

Out of the 2393 cases, 918 (38.4\%) were classified into 224 clusters comprising 2-70 patients per cluster. Among the 918 cluster members, epidemiological links could be confirmed in 340 (37.0\%) patients. In total, $55(2.3 \%)$ patients were healthcare workers; 26 healthcare workers remained unclustered, but 29 healthcare workers belonged to cluster groups. Conventional contact tracing performed before genotyping to identify sources of the reported index cases detected only 73 (3.1\%) patients.

Logistic regression analysis confirmed work in the healthcare sector as strongest predictor for clustering of patients with verified epidemiological links (odds ratio (OR) 3.1, 95\% CI 1.6-5.9), followed by alcoholism (OR 2.3, 95\% CI 1.7-3.2) and sputum smear positivity (OR 1.8, 95\% CI 1.4-2.3). Immigrants were more likely to be cluster nonmembers (OR 0.3, 95\% CI 0.3-0.5). Recent transmission in Hamburg within the 19-year study period was found to be strongly associated with working in a healthcare facility. Although clusters also include many "imported" strains from abroad or regional highly prevalent M. tuberculosis strains with no evident epidemiological connection, routine molecular-epidemiological survey is indispensable to optimising and controlling the effectiveness of TB control strategies in German healthcare settings.

@ERSpublications

Tuberculosis transmission among healthcare workers http://ow.ly/aGn030iGLvF

Cite this article as: Diel R, Niemann S, Nienhaus A. Risk of tuberculosis transmission among healthcare workers. ERJ Open Res 2018; 4: 00161-2017 [https://doi.org/10.1183/23120541.001612017].

Received: Dec 152017 | Accepted after revision: Feb 162018

Copyright $\odot$ ERS 2018. This article is open access and distributed under the terms of the Creative Commons Attribution Non-Commercial Licence 4.0. 


\section{Introduction}

In order to investigate the characteristics of tuberculosis (TB) disease and its transmission in Hamburg, one of the German federal states and the second-largest city in Germany with nearly 1.8 million inhabitants, a prospective epidemiological study was begun in 1997 with the National Reference Center for Mycobacteria in Borstel and the Hamburg Public Health Service working in close collaboration. In this population-based Hamburg fingerprint study, classical epidemiological approaches were combined with molecular biological methods, and genotyping of the Mycobacterium tuberculosis complex strains was performed continuously in Borstel from all available cultures (IS6110 DNA fingerprint and spoligotyping). The resulting typing data of the individual isolates were then compared with one another with regard to agreement (cluster analysis).

DNA fingerprinting denotes molecular typing of a strain of $M$. tuberculosis complex, and ideally addresses the question of whether a single endogenous reactivation of an earlier latent TB infection has developed in a TB patient or whether a "fresh" transfer by an index person has occurred. This can be documented by the occurrence of subsequent TB disease in the contact person caused by a M. tuberculosis agent of the same strain within months or only a few years, with or without evidence of microepidemics ("outbreaks"). The forming of a "cluster", i.e. of a particular M. tuberculosis complex strain affecting at least two different patients, is always the result of a two-stage process: in the first (necessary) stage, an infection occurs by transfer from the diseased index person to the follow-up person (as primary infection or secondary reinfection). In the second step (as a sufficient condition), this latent TB must proceed to the manifest disease.

In Germany the incidence of TB is relatively low. In 1997 (at the beginning of the Hamburg fingerprint study), 9717 cases were recorded by the German Central Committee for the Control of Tuberculosis (DZK) [1]; in 2015, 5865 TB cases (of those 3969 foreign-born, 1533 German-born and 363 TB cases of unknown origin) were reported to the Robert Koch Institute [2], which corresponds to an incidence of 7.3 per 100000 of the residential population.

However, at the same time, the ratio between the respective incidences for foreign-born and German-born residents increased, so that the current TB situation in Germany is predominantly characterised by TB in foreign-born patients. This development has particular impact in the city of Hamburg, where, at the end of 2015, the share of foreign residents in the total population of Hamburg was nearly $15 \%$ (14.7\%) [3], compared the national average of $10.6 \%$ [4]. As early as 2001, TB incidence in the German population had declined to a relatively low level of 8.0 per 100000 inhabitants [5], while the corresponding level of foreign-born residents was 59.6 per 100000 , more than seven times higher than the level of the natives. In 2015 the TB incidence of 10.8 per 100000 in Hamburg was above the national average of 7.3 per 100000 inhabitants [2].

Of particular importance for the targeting of the TB control by public health authorities is the analysis of those cases that are due to "recent transmission". Identifying these makes it possible to identify regionally specific risk groups ahead of the outbreak of disease. The likelihood of identifying cases of recent transmission increases with increasing documentation of cases from the considered "population at risk".

In a preliminary evaluation of the first 6 years of the molecular-epidemiological Hamburg fingerprint study, 848 isolates of TB patients had been genotyped using IS6110 restriction fragment length polymorphism (RFLP) and spoligotyping [6]. At that time, transmission was found to be strongly associated with work in the healthcare professions. Today, the fingerprint study has been ongoing for 19 years. It was our intention to clarify whether the formerly reported results (which were of limited power because of the low number of only 10 genotyped healthcare workers) remain valid and whether genotyping can therefore be recommended for routine use in healthcare settings.

\section{Material and methods}

\section{Methodology of the Hamburg fingerprint study}

The molecular-epidemiological study is embedded in mandatory routine surveillance performed by the public health department according to the legal mandate of the German infectious diseases law (IfSG) and approved by the Hamburg commissioner for data protection. It includes all patients with culture-confirmed TB resident in Hamburg and who thus had to be reported by name according to paragraph 6 of the IfSG.

\section{Epidemiological questions}

Data from patients diagnosed with TB between January 1997 and December 2015 were collected prospectively by trained healthcare personnel using a standardised questionnaire. The following information was obtained from the survey of each patient: sex, date of birth and country of birth, 
nationality, immigration status (if applicable), number of years of residence in Hamburg (or Germany), current address (or whether the patient was homeless), whether the patient was living in a public institution, the type of employment of the patient (in particular with regard to healthcare activities), previous exposure to other persons with TB (in particular within the 6 months before the first appearance of TB-related symptoms) as well as the names of contact persons, in particular the patient's household contacts and any close contacts at the workplace or in community accommodation.

In order to obtain clinical variables, the following data were included: approximate date of the onset of disease (if possible), the date and the reason for the subsequent diagnosis, the time lag between initial presentation and definitive diagnosis, the time interval between the last possible exposure date and the onset of symptoms as well as comorbidities (e.g. diabetes or sarcoidosis and immunosuppressive medication), the time interval between the first environmental assessment and the appearance of symptoms, the nature of the symptoms themselves, the date of the first notification to the public health department according to the IfSG, the results of microbiological investigations and documentation of intravenous drug use and alcohol dependency according to World Health Organization criteria. Extrapulmonary TB disease was defined as TB without signs of lung involvement. When a patient was recognised to be a cluster member, additional interviews were conducted if the patient was available, i.e. still living in Hamburg with a known current location.

\section{Bacterial strains and resistance testing}

All isolates were identified as members of the M. tuberculosis complex, using gene probes (ACCUProbe; Hologic, San Diego, CA, USA) or the GenoType MTBC assay (Hain Lifescience, Nehren, Germany). The differentiation between the species of the $M$. tuberculosis complex was based on genotyping data of each individual isolate [7-9]. Drug susceptibility testing was determined by means of the proportioning method on Löwenstein-Jensen medium and/or by means of the modified proportioning method with the BACTEC MGIT 960 system (Becton Dickinson Microbiology Systems, Cockeysville, MD, USA).

\section{DNA fingerprint analysis}

The extraction of DNA from mycobacterial strains and subsequent IS6110 DNA fingerprinting as well as spoligotyping (spacer oligonucleotide typing) were performed using standardised protocols as described previously [10-12]. The genotyping data were analysed using BioNumerics software (Windows NT version 7.5; Applied Maths, Kortrijk, Belgium) [13].

Clusters were identified when an isolate from one patient delivered an identical DNA fingerprint pattern to that of the isolate from another patient. Since IS6110 was the only effective DNA fingerprint method available in 1997, primary cluster analysis is based on IS6110 DNA fingerprint data. Patients whose isolates had fewer than five identical IS6110 bands were not included in this study, due to the lack of specificity of the method for low-band isolates.

\section{Statistical analysis}

Categorical data were compared using Pearson's Chi-squared test (or Fisher's exact test, when the expected cell sizes were smaller than five). The Wilcoxon rank sum test was used for determining whether the distribution of continuous variables differed between two groups. A two-tailed p-value $<0.05$ was taken as statistically significant. Odds ratios (ORs) and 95\% confidence intervals were calculated using logistic regression analysis (SPSS version 23; IBM, Armonk, NY, USA). As TB patients aged $<16$ years ( $\mathrm{n}=66$ ) were too young to have careers in healthcare and thus could not contribute to the analysis of occupationally transmitted $\mathrm{TB}$, they were excluded from regression analysis.

Variables were included in the final regression model for healthcare work if they changed the OR for healthcare workers by $\geqslant 10 \%$.

\section{Results}

Origin of the examined patients

By December 31, 2015, a total of 3815 patients with TB had been reported to the public health department of Hamburg-Mitte; of those, 2679 patients had been culture-confirmed. The isolates of 2414 patients with culturally confirmed tuberculosis (90.1\%) were available for DNA fingerprint analysis. In 21 $(0.9 \%)$ patients the positive culture could be attributed to laboratory cross-contamination. Therefore, these were excluded from further analysis. Of the 2393 "real" TB patients, 1864 (77.9\%) had pulmonary TB and 529 (22.1\%) patients had extrapulmonary TB only. $984(41.4 \%)$ patients were born in Germany. The remaining 1409 (58.9\%) foreign patients were from 128 different countries, including 170 patients from Turkey (7.1\% of all TB patients) and 156 patients from Afghanistan (6.5\%), but only five patients from Iraq and one patient from Syria. 
Sociodemographic and clinical characteristics

$1470(61.4 \%)$ of all patients were male and 923 (38.6\%) were female; the sex ratio was thus $\sim 1.6: 1$. This relationship did not differ for the 1409 foreign-born patients, of whom 849 (60.3\%) were male and 560 were female (39.7\%). The patients were aged between 9 months and 98 years (mean \pm SD $45.9 \pm 19.4$ years). Of the 1864 patients with pulmonary TB, 921 (49.2\%) were sputum smear-positive at the time of diagnosis, while the others were only culture positive.

Of the M. tuberculosis isolates, 244 (10.2\%) showed resistance to one of the four antituberculous first-line drugs (isoniazid, rifampicin, pyrazinamide and ethambutol). Only 25 (1.0\%) patients were multidrug-resistant (MDR), i.e. resistant to both isoniazid and rifampicin, if not more antituberculous drugs.

\section{Species differentiation}

The species differentiation revealed 2373 (99.2\%) infections with $M$. tuberculosis, 13 infections with $M$. bovis, five infections with $M$. africanum and two infections with M. microti.

\section{Clustering with and without epidemiological confirmation}

The IS6110-based RFLP typing of all 2393 TB cases revealed 224 clusters, comprising 918 (38.4\%) patients, of whom 422 were foreign born (17.6\% of all TB cases included). The largest cluster identified in this study consisted of 70 patients, including 29 (41.4\%) positive sputum outcomes. A detailed analysis of this cluster was published in 2013 in PLoS Medicine [14].

Among the 224 clusters, 97 (43.3\%) were found in which at least one transmission from cluster member to another was epidemiologically confirmed. 220 cluster members were infected by index patients $(24.0 \%$ of all cluster members). If the respective index persons were included, 340 patients $(37.0 \%$ of all cluster members) were from a confirmed epidemiological context. There was no transmission of $M$. bovis and $M$. microti strains.

\section{Healthcare workers and transmission risks}

Of the 2393 patients registered in the Hamburg fingerprint study up until the end of 2015, a total of 55 patients were employed in healthcare professions. 29 of these were part of clusters and 26 healthcare workers were non-cluster patients (figure 1). In 15 of the 29 cluster members, one patient had TB transmission to another healthcare worker $(27.3 \%)$ and in one case a healthcare worker transmitted his M. tuberculosis strain to two consecutive patients (1.8\%). Two patients of the same cluster each transmitted MDR-TB to another healthcare worker (figure 2). One healthcare worker infected a family member $(1.8 \%)$. For $12(21.8 \%)$ of the afflicted healthcare worker who were cluster members, there was no evidence of $M$. tuberculosis transmission within the study period. In total, two healthcare workers had MDR-TB.

\section{Risk factors for clustering and recent transmission}

Our work began with the performance of univariate analysis to identify significant differences between the 918 clustered patients and the 1475 non-clustered patients (table 1).

In that study, the clustered patients were significantly more frequently homeless (124 out of 918, 13.5\%) than non-cluster members (93 out of $1475,6.3 \%$; $\mathrm{p}<0.001$ ) and more often dependent on intravenously delivered drugs $(\mathrm{p}<0.001)$. In addition, they were more frequently sputum smear-positive $(\mathrm{p}<0.001)$ and males were more than twice as likely as females to be smear-positive. In a Wilcoxon rank sum test there was a significant difference $(\mathrm{p}<0.001)$ in the age distribution between the two groups, since cluster patients were 5 years younger (mean $\pm \mathrm{SD} 43.9 \pm 17.4$ years versus $47.7 \pm 20.3$ years). Healthcare workers were significantly more frequently cluster members $(29$ (3.2\%) out of 918 versus $26(1.8 \%)$ out of $1475, \mathrm{p}<0.05)$.

Independent risk factors for confirmed recent transmission were then determined by logistic regression (table 2). Here, employment in the healthcare sector was the strongest predictor of a transmission (OR 3.07, $\mathrm{p}=0.001$ ), followed by alcohol abuse (OR 2.3, $\mathrm{p}<0.001)$ and a positive sputum smear (OR 1.8, $\mathrm{p}<0.001$ ). TB patients were more frequently involved in recent transmission with decreasing age (OR 0.96 , $\mathrm{p}<0.001)$, i.e. an increase in age of TB patients by 1 year was associated with a reduction in the chance of transmission by $4 \%$ compared with the previous year. However, unemployment, drug dependence, male sex, resistance against any antituberculous drug or homelessness did not represent an independent risk of being part of an identified transmission chain. In contrast, foreign-born citizens were not only rarely represented in clusters compared to German-born citizens; they were also significantly less involved in identified cases of $M$. tuberculosis transmission (OR 0.36, $\mathrm{p}<0.001$ ). 


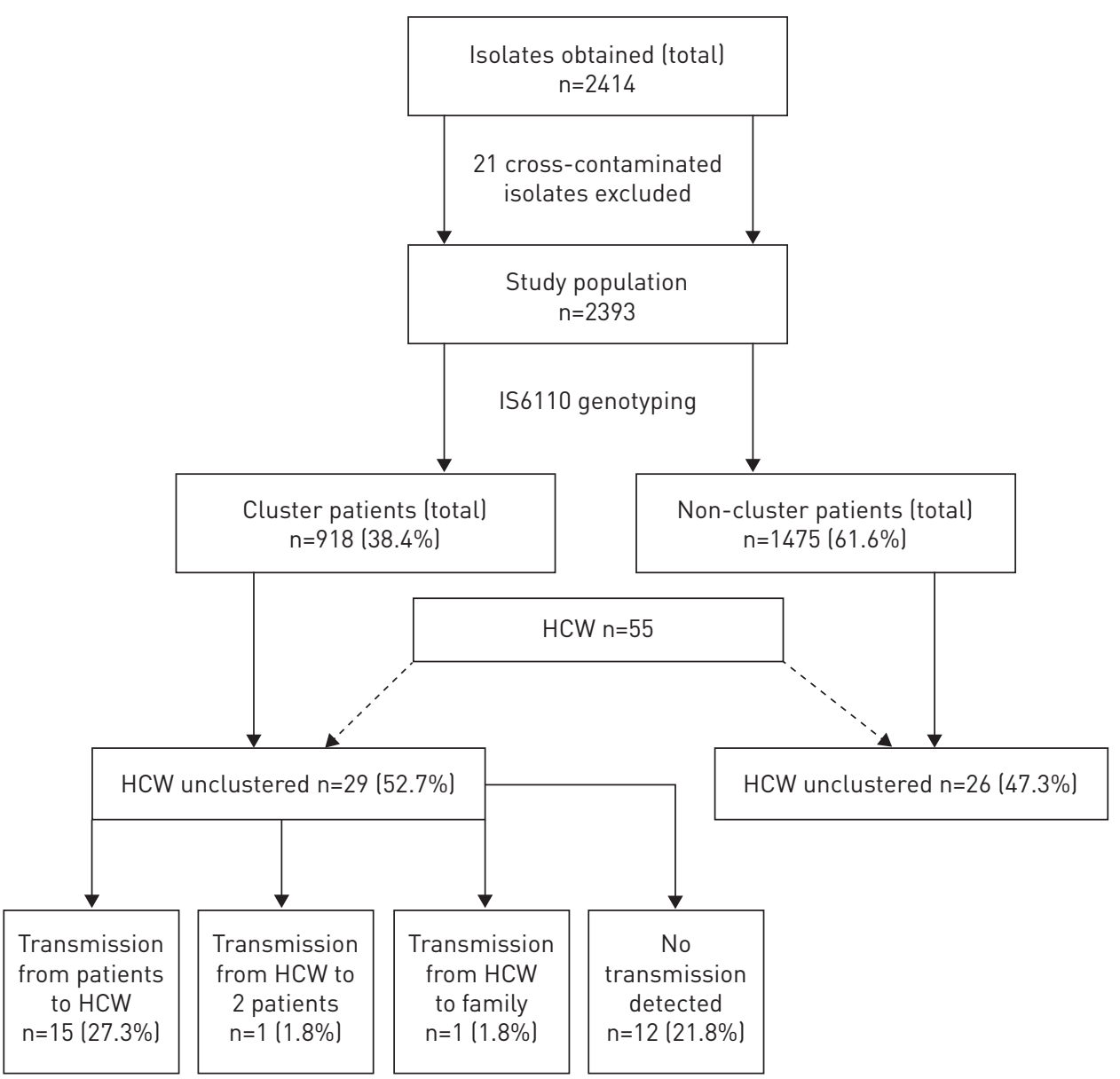

FIGURE 1 Clustering of healthcare workers (HCWs) and patterns of transmission.

\section{Conventional contact tracing}

On average, each of the 1864 patient with culture-confirmed and thus potentially infectious pulmonary TB had 5.8 \pm 8.7 contact individuals (median 4, range $0-77$ ). The number of contact persons investigated did not differ significantly between German-born $(n=1654$, mean \pm SD $8.0 \pm 10.8)$ and foreign-born patients $(\mathrm{n}=1359$, mean \pm SD $6.0 \pm 8.9 ; \mathrm{p}=0.13)$.

Only $73(3.1 \%)$ out of the total 2393 patients were diagnosed directly by conventional contact tracing, or after the established period of 9 months within which a second chest radiograph examination has to be performed. Of those, only two were healthcare workers. Of these 73 "contact individuals", 51 (69.9\%) were

a)

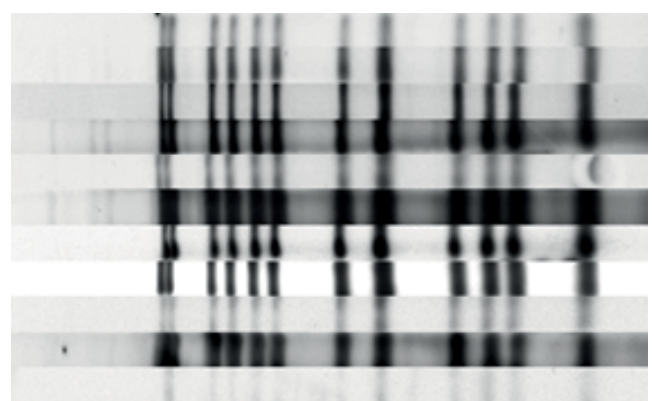

b)

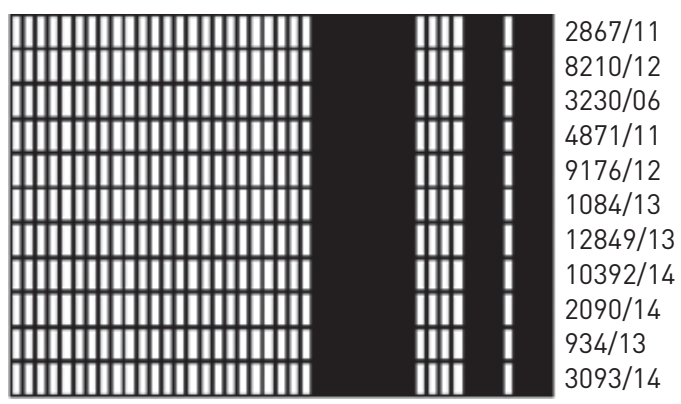

FIGURE 2 a) IS6110 restriction fragment length polymorphism (RFLP) and b) spoligotyping patterns of a multidrug-resistant tuberculosis (MDR-TB) cluster. Identical IS6110 RFLP and spoligotyping patterns of the isolates of 11 MDR-TB cluster members. Two male prostitutes (strain no. 8210/12 and no. 9167/12) each infected one healthcare worker (strain no. $2090 / 14$ and 10392/14) in a facility for street sex workers. 


\begin{tabular}{|c|c|c|c|c|}
\hline & Non-cluster group & Cluster group & All patients & p-value \\
\hline Patients & 1475 & 918 & 2393 & \\
\hline Age years & $47.3 \pm 19.5$ & $37.6 \pm 16.5$ & $45.9 \pm 19.4$ & $<0.001$ \\
\hline AIDS & $110(7.5)$ & $65(7.1)$ & 175 (7.3) & NS \\
\hline Resistance to any drug & 165 (11.2) & $79(8.6)$ & $244(10.2)$ & 0.04 \\
\hline Beijing genotype & $99(6.7)$ & $61(6.6)$ & $160(6.7)$ & NS \\
\hline Foreign-born & $987(66.9)$ & $422(46.0)$ & $1409(58.9)$ & $<0.001$ \\
\hline Male/female & $851 / 624$ (57.7/42.3) & $619 / 299(67.4 / 32.6)$ & $1470 / 923(61.4 / 38.6)$ & $<0.001$ \\
\hline Drug abuse & $36(2.4)$ & $90(9.8)$ & $126(5.3)$ & $<0.001$ \\
\hline Alcohol abuse & $121(8.2)$ & $313(34.1)$ & $434(18.1)$ & $<0.001$ \\
\hline Homelessness & 93 (6.3) & $124(13.5)$ & $217(9.1)$ & $<0.001$ \\
\hline Sputum smear positivity & $474(32.1)$ & 447 (48.7) & $921(38.5)$ & 0.001 \\
\hline Previous history of TB & $165(11.2)$ & $98(10.7)$ & $263(11.0)$ & NS \\
\hline Unemployment & $371(25.2)$ & $427(46.5)$ & 798 (33.3) & $<0.001$ \\
\hline Healthcare worker & $26(1.8)$ & $29(3.2)$ & $55(2.3)$ & 0.027 \\
\hline
\end{tabular}

cluster members, each with a verified epidemiological link to a source case. The rate here was significantly higher in German-born than in foreign-born patients ( 41 out of 49 versus 10 out of $24, \mathrm{p}<0.001$ ). In the other $22(30.1 \%)$ cases, the disease must be regarded as coincidental and due to infection by earlier exposure to a carrier other than the suspected index person. In addition, only $26(51.1 \%)$ out of the 51 confirmed contacts were established within the customary period of 9 months. The remaining 25 patients (of those one of the two traced healthcare workers) became ill outside that period and sought medical help due to typical symptoms.

\section{Imported clusters}

Therefore, for each individual cluster the challenge of a precise as well as a subtle epidemiological analysis always arises. Clusters may arise by chance with member patients never having had contact with one another. Table 3 shows five examples of clusters of foreign-born residents of Hamburg from different years, where it was obvious at the outset that transmission could not have occurred recently; the clear assumption is that the cluster members had been infected by a highly prevalent regional strain in their respective home country before "importing" it to Hamburg.

In cluster A, which consists of two Turkish citizens, the first patient migrated to Hamburg in July 1997 and presented a cervical lymph node TB caused by reactivation of a formerly latent $M$. tuberculosis infection. The second patient, whose disease was diagnosed 4 weeks later, showed the first symptoms of his infectious pulmonary $\mathrm{TB}$ at about the same time. Because the lymph node $\mathrm{TB}$ must be considered noninfectious, and given the nearly simultaneous onset of their TB disease, transmission could not be

TABLE 2 Results of multiple logistic regression of risk factors for epidemiologically confirmed transmission in tuberculosis cluster patients

\begin{tabular}{lcc} 
& Odds ratio $(95 \%$ CI) & p-value \\
\hline Healthcare worker & $3.07(1.59-5.92)$ & 0.001 \\
Alcohol abuse & $2.30(1.66-3.18)$ & $<0.001$ \\
Sputum smear positivity & $1.79(1.39-2.32)$ & $<0.001$ \\
Drug addiction (i.v.) & $1.12(0.71-1.78)$ & 0.62 \\
Unemployment & $1.18(0.88-1.60)$ & 0.27 \\
Male & $1.23(0.92-1.65)$ & 0.16 \\
Any drug resistance & $0.86(0.55-1.36)$ & 0.52 \\
Homelessness & $0.995(0.64-1.42)$ & 0.98 \\
Age & $0.96(0.95-0.97)$ & $<0.001$ \\
Foreign born & $0.36(0.27-0.48)$ & $<0.001$ \\
\hline
\end{tabular}




\section{TABLE 3 Examples of clusters "imported" to Hamburg}

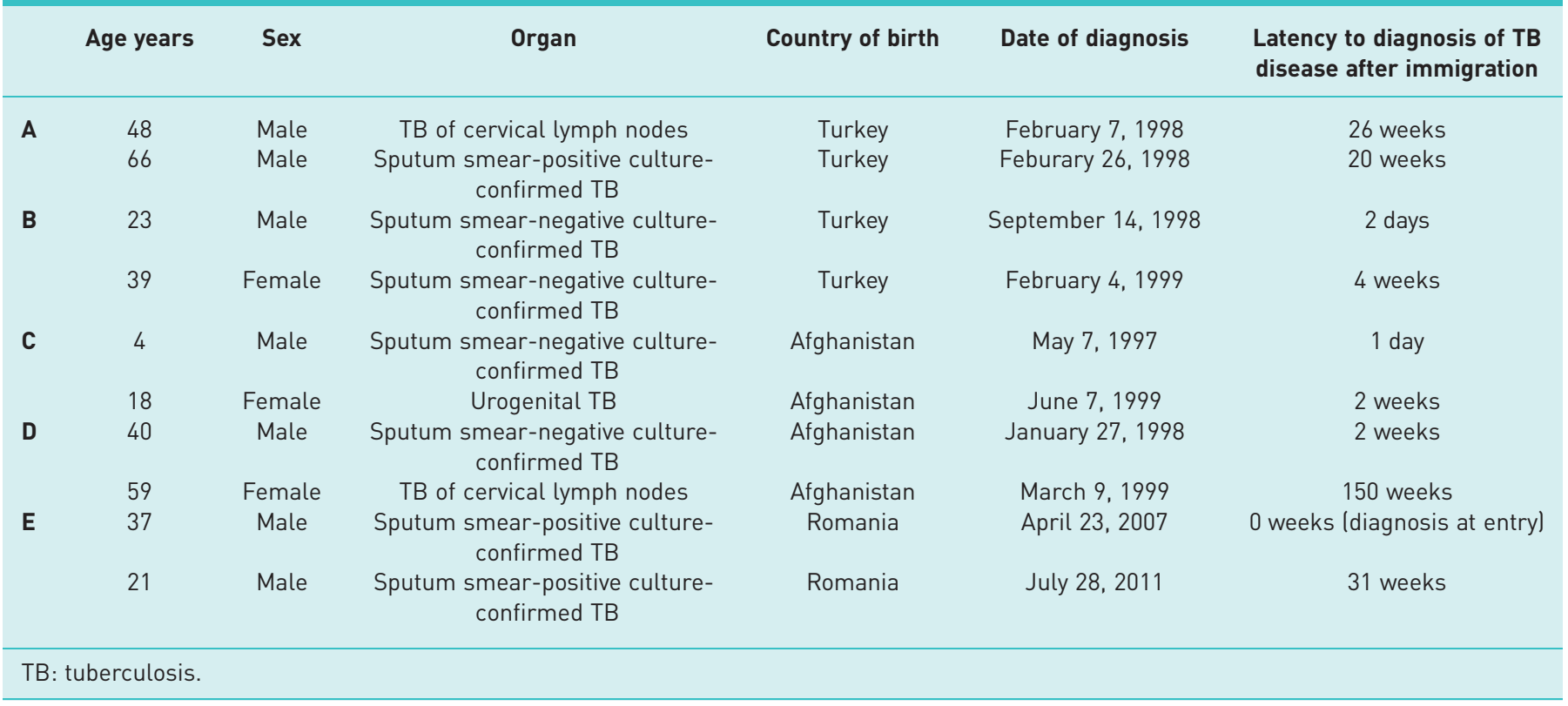

assumed or proven. The subsequent epidemiological analysis did not indicate any previous contact between the two patients (not even in their home country); however, the two had grown up in the same regional district in Turkey.

In cluster $\mathrm{B}$, the male patient had already developed a sputum smear-negative, but culture-confirmed pulmonary TB when he came to Hamburg, where he was treated immediately. The female cluster member showed an identical strain, although she entered Germany just 4 months later and had never met the male cluster member whose sputum cultures had become negative in the meantime. Thus, M. tuberculosis transmission between the two patients could be excluded a priori.

Cluster $\mathrm{C}$ had a similar constellation with a still more striking temporal difference to the (theoretically) possible index person, whose TB disease had in fact been treated long before the second cluster member arrived in Hamburg.

In cluster D, the Afghan patient with cervical lymph node TB had arrived in Hamburg almost 2 years before the pulmonary TB patient; the latter developed his TB during the entry process and thus could not be considered to be the index patient.

In cluster E, the first patient, whose pulmonary TB had already been diagnosed in Romania, was treated immediately after arrival in Hamburg without making contact with any compatriots. The patient returned to Romania after 6 months' treatment in 2008. The second member of the cluster had been latently infected with $M$. tuberculosis as a 7-year-old child in his home country, and travelled as an adult to Germany in November 2011. There he suffered progression to TB disease after $<8$ months.

\section{Discussion}

The results of the population-based Hamburg fingerprint study deliver a detailed picture of TB epidemiology over a period of 19 years and demonstrate that, even in a country with a low TB incidence, a large proportion of the TB disease may be due to recent transmission. However, the proportion of recent transmissions confirmed by epidemiological data corresponds to only a subset of the identified cluster patients. Studies performed elsewhere with molecular TB characterisation have shown that validated epidemiological links to another cluster member can be found in only $25-64 \%$ of patients in clusters [15-18].

Even though intensive epidemiological interview techniques and careful geographical mapping of the dwelling places of cluster members were employed, transmission was determined in only $37 \%$ of the patients grouped in a cluster. Our examples of the five "imported" clusters described may partially explain why foreign-born patients do not contribute much to the spread of $M$. tuberculosis strains in their new 
German domicile, although they constitute the majority $(n=1409,58.9 \%)$ of TB patients and contribute nearly half $(48.9 \%)$ of the cluster members.

In contrast, where transmission can be epidemiologically verified, it appears to be at least partially promoted by conditions specific to our location. In our study, several risk factors for such clusters were identified using univariate analysis that, beside sputum positivity as prerequisite of rapid infection, may be seen as the negative characteristics of this seaport metropolis: alcohol abuse, unemployment and intravenous drug use. One benevolent factor stands out, that of being a healthcare worker. Significantly, the multivariate analysis showed that employment in the healthcare sector was the strongest risk factor for recent $M$. tuberculosis transmission in Hamburg. This observation confirms the results of the preliminary study that had examined the data collected up to 2002 [6]. It may be explained by the great number of unexpected and initially undiagnosed TB cases in Hamburg healthcare facilities.

Of note, population-based information on the impact of TB transmission in healthcare settings in light of decreasing incidence in most Western industrialised countries is sparse, and notably, since the publication of our preliminary study only very little molecular-epidemiological work has dealt with that issue. FoK et al. [19], in their systematic review on risk factors for clustering analysed 36 population-based studies from 17 countries published between 1995 and 2005, but transmission in healthcare setting was not included in most of these studies as a variable of interest.

In the meantime, KAMPER-JøRGENSEN et al. [20] reported $M$. tuberculosis transmission from a patient to a nurse following an extremely short exposure by retrospective evaluation of the Danish TB Subtyping Database, comprising $>6000$ DNA patterns from TB patients nationwide. NoDIEVA et al. [21] demonstrated that direct epidemiological links in hospitals verified by IS6110 RFLP contributes to almost one-third (32\%) of all MDR Beijing cases found in Eastern Europe. Appelgren et al. [22] recently described a case of transmission of pre-extensively drug-resistant Beijing isolate to a French healthcare worker, underscoring the risk of healthcare-associated contamination in low-incidence countries and the importance of molecular tools of TB care.

Although the majority of TB diseases in healthcare workers in our Hamburg study resulted from reactivation of latent $\mathrm{TB}$ infection with an unclear origin, more than a quarter (15 out of 51 healthcare workers, $27.3 \%$ ) could be shown to have acquired the underlying infections within the healthcare system. Thus, our longitudinal study underlines again that the transmission of TB in healthcare facilities may be of great significance. Furthermore, the number of unidentified, recently transmitted and occupationally acquired cases may be high, as only those healthcare workers were investigated who were residents of Hamburg proper. Healthcare workers who serve in Hamburg but live in the surrounding communities were not included.

In conclusion, the results of our study demonstrate that even in countries with low $\mathrm{TB}$ incidence healthcare workers are at particular risk of recent TB transmission. As conventional contact tracing plays only a minor role in the detection of TB transmission, continuous insight into the effectiveness of control measures in healthcare facilities can only be provided by genotyping of $M$. tuberculosis strains. Therefore, its practice should be extended beyond the currently limited number of federal states to establish it a national standard of care in Germany.

Author contributions: R. Diel conceived and designed the study, acquired, analysed and interpreted the data, and drafted and revised the article. S. Niemann performed fingerprinting of the Mycobacterium tuberculosis isolates and built the clusters, drafted and revised the article, and gave final approval of the version to be published. A. Nienhaus analysed and interpreted the data, drafted and revised the article, and gave final approval of the version to be published.

Conflict of interest: None declared.

\section{References}

1 Loddenkemper R, Sagebiel S, Meyer C, et al. Die Tuberkulosesituation in Deutschland 1998. [The state of tuberculosis in Germany 1998]. Pneumologie 2000; 54: 324-332.

2 Robert Koch-Institut. Bericht zur Epidemiologe der Tuberkulose für Deutschland 2015. Berlin, Robert Koch-Institut, 2016

3 Statistical Office for Hamburg and Schleswig Holstein. Bevölkerung in Hamburg 2015. [Population in Hamburg 2015]. www.statistik-nord.de/fileadmin/Dokumente/Presseinformationen/SI16_131.pdf

4 Statistisches Bundesamt. Bevölkerungsfortschreibung 2015 [Population Update 2015]. Wiesbaden, Germany, Statistisches Bundesamt. www.destatis.de/DE/ZahlenFakten/GesellschaftStaat/Bevoelkerung/Bevoelkerungsstand/ Glossar/FortschreibungdesBevoelkerungsstandes.html

5 Hygiene Institut Hamburg. Meldepflichtige Infektionskrankheiten in Hamburg 2001. Jahresbericht [Notifiable Infectious Diseases in Hamburg 2001. Annual Report]. Hamburg, Germany, Hygiene Institut Hamburg, 2001.

6 Diel R, Seidler A, Nienhaus A, et al. Occupational risk of tuberculosis transmission in a low incidence area. Respir Res 2005; 6: 35. 
7 Diel R, Rüsch-Gerdes S, Niemann S. Molecular epidemiology of tuberculosis among immigrants in Hamburg, Germany. J Clin Microbiol 2004; 42: 2952-2960.

8 Niemann S, Rüsch-Gerdes S, Richter E. IS6110 fingerprinting of drug-resistant Mycobacterium tuberculosis strains isolated in Germany during 1995. J Clin Microbiol 1997; 35: 3015-3020.

9 Wirth T, Hildebrand F, Allix-Béguec C, et al. Origin, spread and demography of the Mycobacterium tuberculosis complex. PLoS Pathog 2008; 4: e1000160.

10 Niemann S, Supply P. Diversity and evolution of Mycobacterium tuberculosis: moving to whole-genome-based approaches. Cold Spring Harb Perspect Med 2014; 4: a021188.

11 Niemann S, Richter E, Rüsch-Gerdes S. Stability of Mycobacterium tuberculosis IS6110 restriction fragment length polymorphism patterns and spoligotypes determined by analyzing serial isolates from patients with drug-resistant tuberculosis. J Clin Microbiol 1999; 37: 409-412.

12 Kamerbeek J, Schouls L, Kolk A, et al. Simultaneous detection and strain differentiation of Mycobacterium tuberculosis for diagnosis and epidemiology. J Clin Microbiol 1997; 35: 907-914.

13 van Embden JDA, Cave MD, Crawford JT, et al. Strain identification of Mycobacterium tuberculosis by DNA fingerprinting: recommendations for a standardized methodology. J Clin Microbiol 1993; 31: 406-409.

14 Roetzer A, Diel R, Kohl TA, et al. Whole genome sequencing versus traditional genotyping for investigation of a Mycobacterium tuberculosis outbreak: a longitudinal molecular epidemiological study. PLoS Med 2013; 10: e1001387.

15 Alland D, Kalkut GE, Moss AR, et al. Transmission of tuberculosis in New York City. An analysis by DNA fingerprinting and conventional epidemiologic methods. N Engl J Med 1994; 330: 1710-1716.

16 Anh DD, Borgdorff MW, Van LN, et al. Mycobacterium tuberculosis Beijing genotype emerging in Vietnam. Emerg Infect Dis 2000; 6: 302-305.

17 Bauer J, Yang Z, Poulsen S, et al. Results from 5 years of nationwide DNA fingerprinting of Mycobacterium tuberculosis complex isolates in a country with a low incidence of M. tuberculosis infection. J Clin Microbiol 1998; 36: 305-308.

18 Dale JW, Nor RM, Ramayah S, et al. Molecular epidemiology of tuberculosis in Malaysia. J Clin Microbiol 1999; 37: 1265-1268.

19 Fok A, Numata Y, Schulzer M, et al. Risk factors for clustering of tuberculosis cases: a systematic review of population-based molecular epidemiology studies. Int J Tuberc Lung Dis 2008; 12: 480-492.

20 Kamper-Jørgensen Z, Lillebaek T, Andersen AB. Occupational tuberculosis following extremely short exposure. Clin Respir J 2009; 3: 55-57.

21 Nodieva A, Jansone I, Broka L, et al. Recent nosocomial transmission and genotypes of multidrug-resistant Mycobacterium tuberculosis. Int J Tuberc Lung Dis 2010; 14: 427-433.

22 Appelgren A, Morquin D, Dufour S, et al. Investigation of pre-XDR Beijing Mycobacterium tuberculosis transmission to a healthcare worker in France, 2016. J Hosp Infect 2017; 97: 414-417. 